\title{
Population Pharmacokinetic Analysis from First-in-Human Data for HDAC Inhibitor, REC-2282 (AR-42), in Patients with Solid Tumors and Hematologic Malignancies: A Case Study for Evaluating Flat vs. Body Size Normalized Dosing
}

\author{
Sophia Liva ${ }^{1} \cdot$ Min Chen ${ }^{1} \cdot$ Amir Mortazavi $^{2,3} \cdot$ Alison Walker $^{3,4} \cdot$ Jiang Wang $^{3} \cdot$ Kristin Dittmar $^{5} \cdot$ Craig Hofmeister $^{6}$. \\ Christopher C. Coss ${ }^{1,3} \cdot$ Mitch A. Phelps ${ }^{1,3}$
}

Accepted: 12 September 2021 / Published online: 7 October 2021

(c) The Author(s) 2021

\begin{abstract}
Background and Objectives REC-2282 is a novel histone deacetylase inhibitor that has shown antitumor activity in in vitro and in vivo models of malignancy. The aims of this study were to characterize the population pharmacokinetics of REC2282 (AR-42) from the first-in-human (NCT01129193) and phase I acute myeloid leukemia trials (NCT01798901) and to evaluate potential sources of variability. Additionally, we sought to understand alternate body size descriptors as sources of inter-individual variability (IIV), which was significant for dose-normalized maximum observed concentration and area under the concentration-time curve (AUC).

Methods Datasets from two clinical trials were combined, and population pharmacokinetic analysis was performed using NONMEM and R softwares; patient demographics were tested as covariates.

Results A successful population pharmacokinetic model was constructed. The pharmacokinetics of REC-2282 were best described by a two-compartment model with one transit compartment for absorption, first-order elimination and a proportional error model. Fat-free mass (FFM) was retained as a single covariate on clearance (CL), though it explained $<3 \%$ of the observed variability on CL. Tumor type and formulation were retained as covariates on lag time, and a majority of variability, attributed to absorption, remained unexplained. Computed tomography (CT)-derived lean body weight estimates were lower than estimated lean body weight and fat-free mass measures in most patients. Analysis of dose-normalized AUC vs. body size descriptors suggests flat dosing is most appropriate for REC-2282.

Conclusions FFM was identified as a significant covariate on CL; however, it explained only a very small portion of the IIV; major factors contributing significantly to REC-2282 pharmacokinetic variability remain unidentified.
\end{abstract}

Sophia Liva and Min Chen contributed equally to this manuscript.

Christopher C. Coss

coss.16@osu.edu

Mitch A. Phelps

phelps.32@osu.edu

1 Division of Pharmaceutics and Pharmacology, College of Pharmacy, The Ohio State University, Columbus, $\mathrm{OH}$, USA

2 Division of Medical Oncology, Department of Internal Medicine, The Ohio State University, Columbus, OH, USA
3 Comprehensive Cancer Center, The Ohio State University, Columbus, $\mathrm{OH}$, USA

4 Division of Hematology, Department of Internal Medicine, College of Medicine, The Ohio State University, Columbus, $\mathrm{OH}$, USA

5 Department of Radiology, Wexner Medical Center, Columbus, OH, USA

6 Division of Hematology, Department of Hematology and Medical Oncology, Winship Cancer Institute of Emory University, Atlanta, GA, USA 


\section{Key Points}

Population pharmacokinetic analysis of REC-2282 (AR-42) demonstrates high pharmacokinetic variability, which may be a function of variable oral absorption

The present analysis suggested FFM does explain a very small portion of IIV $(<3 \%)$, and this supports choice of flat dosing, as the minimal decrease in pharmacokinetic variability utilizing body size-normalized dosing would not justify this dosing strategy

\section{Introduction}

Histone deacetylases (HDACs) are overexpressed in a variety of cancers, and HDAC inhibitors are an expanding class of anti-cancer therapeutics that induce growth arrest, differentiation and apoptosis of malignant cells [1, 2]. REC-2282 (also known as AR-42 and referred to as such from this point forward) is a novel HDAC inhibitor that exhibits antitumor activity in in vitro and in vivo models of malignancy, and similar to other pan HDAC inhibitors, suppresses tumor cell growth via a variety of mechanisms. Additionally, REC2282 has shown promising activity in preclinical models of neurofibromatosis type 2 (NF2) [3]. Interestingly, from the first-in-human (FIH) study, the median progression-free survival for solid tumors was 3.6 months compared to 9.1 months in patients with NF2 and meningiomas [4, 5]. In agreement with these clinical findings, an informatics-based artificial intelligence (AI) approach independently suggested REC-2282 would be effective in NF2 [6]. REC-2282 is currently under clinical investigation for vestibular schwannoma and meningiomas (NCT02282917).

In preclinical studies, oral bioavailability was estimated at $26 \%$ and $100 \%$ in mice and rats, respectively (unpublished data). REC-2282 was highly bound to plasma proteins in mouse plasma (96\%). Renal clearance was a minor pathway of elimination, as $1.4 \%$ and $5 \%$ of the dose was recovered in mouse and rat urine, respectively [7]. Preliminary phase 1 data from the first-in-human study with orally administered, flat-dosed REC-2282 revealed a coefficient of variation $(\mathrm{CV})$ ranging from 36 to $76 \%$ among dose levels in apparent oral clearance and $24-66 \%$ in peak concentrations [2]. The maximum tolerated dose (MTD) was found to be $40 \mathrm{mg}$ and 60 mg for patients with hematologic and solid tumor malignancies, respectively, both dosed three times weekly for 3 weeks followed by 1 week off in a 28-day cycle. Observed dose-limiting toxicities included grade 4 thrombocytopenia, febrile neutropenia and grade 3 neutropenia with infection and grade 4 psychosis [2, 4]. Among the 27 patients enrolled, clinical response varied from minimal response to progressive disease, and dose-dependent cytopenias were the most common adverse event. The observed wide inter-individual pharmacokinetic variability (IIV) [8] and variability in clinical response is commonly observed with many anticancer therapies, especially with oral drugs, often requiring dose reductions or termination of therapy $[9,10]$. Typical strategies for individualizing doses include bodysize normalization, though the benefits of this approach have been challenged repeatedly [11-16]. Alternatively, flat dosing is the most convenient and most easily managed dosing approach, especially with orally administered tablet or capsule formulations in fixed dosage strengths. However, the choice of flat vs. body size-normalized dosing, especially within a first-in-human study, should be evaluated to ensure the choice provides acceptable variability in exposures within the targeted patient population.

The primary objectives of the current study were to describe the population pharmacokinetics of REC-2282 using pooled data from NCT01129193, the first-in-human study in solid tumors and hematologic malignancies, and NCT01798901, a phase 1 study in acute myeloid leukemia, and identify covariates that explain portions of IIV in plasma pharmacokinetics. In particular, we explored various body size descriptors as potential sources of IIV. As part of this analysis, we also wanted to evaluate whether or not the use of body size-normalized dosing, as opposed to flat dosing, could further reduce observed pharmacokinetic variability.

\section{Methods}

\subsection{Study Design, Drug Treatment and Patient Enrollment}

The details of trial design concerning the clinical trials included in our analysis have been previously described [2, $4,5,17]$. These studies were approved by The Ohio State University Institutional Review Board and conducted in accordance with the Helsinki Declaration of 1975, as revised in 1983. Patients enrolled in both trials were administered REC-2282 orally three (or four) times weekly, Monday, Wednesday, (Thursday) and Friday in 28-day cycles with 3 weeks of dosing followed by a 7-day off-treatment period (Table 1). Blood samples were collected from a total of 57 subjects enrolled on OSU09102 (NCT01129193) and OSU11130 (NCT01798901) including times points of predose, $(0.25), 0.5,1,1.5,2,4,8,10$ and $24 \mathrm{~h}$ post-dose on either day 1, day 5 and/or day 19 . 


\subsection{Pharmacokinetic Sample Analysis}

Plasma concentrations of REC-2282 were determined using previously described bioanalytical methodology [2] and are summarized in Supplemental Table 1.

\subsection{Pharmacokinetic Modeling and Covariate Analysis}

Pharmacokinetic data were available from 55 subjects, from day 1 , day 5 and/or day 19. REC-2282 plasma concentrations were available. Population pharmacokinetic models were developed using NONMEM, version 7.3, implementing the first-order conditional estimation method with interaction (FOCE-I) [18]. R (version 3.3.1; http://www.r-proje ct.org) and the Xpose package (version 4.5.3; http://xpose. sourceforge.net) were used for visual diagnostics.

REC-2282 concentration data were evaluated with various compartmental models using lag time (ALAG) and transit models to describe the observed apparent delay in REC2282 systemic absorption [19]. IIV was estimated using an exponential error model. Residual variability $(\varepsilon)$ was described with an additive error model for log-transformed data. Tested covariates included age, sex, tumor type, trial, lean body weight (LBW), fat-free mass (FFM), body mass index (BMI), total body weight (TBW), body surface area (BSA) and measured lean body weight (MLBW) by CTderived calculation [20]. Tumor type and trial were evaluated as dichotomous variables for solid vs. heme tumor and OSU09102 vs. OSU11130, respectively. LBW, FFM, BMI and CT based body composition factors were calculated as noted below. Continuous covariates were normalized using population median values, and a power model was used during the covariate model construction:

$\theta_{i}=\theta_{P} \times\left(\frac{\mathrm{COV}}{\text { Median }}\right)^{\theta_{C O V}} \times e^{\eta_{i}}$

where $\theta_{i}$ is the estimated value of a parameter for individual $i, \theta_{P}$ is the estimated typical value of the parameter in the population, $\mathrm{COV}$ is the individual value of the particular covariate under investigation, Median is the median value of the covariate in the study population, $\theta_{\mathrm{COV}}$ is the estimated covariate coefficient determining its effect, and $e^{\eta i}$ is the estimated individual variation between $\theta_{i}$ and $\theta_{p}$ not accounted for by the covariate, whereby the $\eta_{i}$ s in the population are approximately log-normally distributed with a variance of $\omega^{2}$ and mean of zero. For categorical covariates, the following function was used:

$\theta_{i}=\theta_{P} \times\left(1+\theta_{\mathrm{COV}} \times \mathrm{COV}\right) \times e^{\eta_{i}}$

Covariates having a significant influence $(P<0.05)$ were added in a forward stepwise manner until no further
Table 1 Dosing regimens of REC-2282 summarized by dose level and clinical trial

\begin{tabular}{lll}
\hline Dosing regimen & \multicolumn{2}{l}{$\begin{array}{l}\text { Dosing regimen of AR-42 by } \\
\text { clinical trial }\end{array}$} \\
\cline { 2 - 3 } & OSU09102 & OSU11130 \\
\hline No. & 44 & 13 \\
$20 \mathrm{mg}$ (Day 1, 3, 5) $(\%)$ & $3(5.3)$ & $3(5.3)$ \\
$30 \mathrm{mg}$ (Day 1, 3, 5) $(\%)$ & $3(5.3)$ & - \\
$40 \mathrm{mg}$ (Day 1, 3, 5) $(\%)$ & $16(28.1)$ & $3(5.3)$ \\
$40 \mathrm{mg}$ (Day 1,3, 4, 5) $(\%)$ & - & $7(12.3)$ \\
$50 \mathrm{mg}$ (Day 1, 3, 5) $(\%)$ & $7(12.3)$ & - \\
$60 \mathrm{mg}$ (Day 1, 3, 5) $(\%)$ & $12(21.1)$ & - \\
$70 \mathrm{mg}$ (Day 1, 3, 5) $(\%)$ & $1(1.8)$ & - \\
$80 \mathrm{mg}$ (Day 1, 3, 5) $(\%)$ & $2(3.5)$ & - \\
\hline
\end{tabular}

Doses were administered the first 3 weeks, Monday, Wednesday, (Thursday) and Friday within a 4-week (28-day) cycle

reduction in objective function was observed. Backward elimination was then performed using a $P$ value of 0.01 .

The final population pharmacokinetic model was evaluated for accuracy and stability via bootstrap resampling. Model parameters were estimated, and $95 \%$ confidence intervals of the bootstrap replicates were compared with parameter estimates from the final pharmacokinetic model. Model-based simulation was then performed to evaluate predictive performance of the final model using visual predictive checks (VPCs) from 1000 simulations [21]. Simulation was also performed to explore how body size-normalized dosing might have impacted IIV.

\subsection{Body Size and Body Composition Assessments}

The patient characteristics that were collected and evaluated as potential covariates were: age, body weight, BSA, BMI, sex, trial, tumor type, LBW and MLBW by CT. LBW was calculated using TBW and height [22]:

$\mathrm{LBW}(\mathrm{kg})=1.10 \times \mathrm{TBW}(\mathrm{kg})-120 \times\left[\frac{\mathrm{TBW}}{\operatorname{height}(\mathrm{cm})}\right]^{2}($ males $)$

$\mathrm{LBW}(\mathrm{kg})=1.07 \times \mathrm{TBW}(\mathrm{kg})-148 \times\left[\frac{\mathrm{TBW}}{\text { height }(\mathrm{cm})}\right]^{2}($ females $)$

FFM was calculated using TBW and BMI [23]:

$\mathrm{FFM}(\mathrm{kg})=\frac{9.27 \times 103 \times \mathrm{TBW}(\mathrm{kg})}{6.68 \times 103+216 \times \mathrm{BMI}}($ males $)$

$\mathrm{FFM}(\mathrm{kg})=\frac{9.27 \times 103 \times \mathrm{TBW}(\mathrm{kg})}{8.78 \times 103+244 \times \mathrm{BMI}}($ females $)$ 
BMI [24] and BSA [25] were calculated as previously described, using the following formulas:

$\operatorname{BSA}\left(\mathrm{m}^{2}\right)=\sqrt{\frac{\text { height }(\mathrm{cm}) \times \text { weight }(\mathrm{kg})}{3600}}$

$\mathrm{BMI}=\frac{\text { weight }(\mathrm{kg})}{\operatorname{height}(\mathrm{m})^{2}}$

CT images at specific lumbar landmarks correlate to whole-body skeletal muscle in healthy adults [26, 27]. Patients who received a CT scan within 30 days of their first cycle, and therefore pharmacokinetic assessments, were evaluated for skeletal muscle area at the third lumbar vertebra (L3) [20]. Images were analyzed for cross-sectional area (CSA) $\left(\mathrm{cm}^{2}\right)$ using Slice-O-Matic software V4.3 (Tomovision, Montreal, Quebec, Canada), and density was quantified in Hounsfield units (HU) assigned to each image pixel relative to reference values for air $(-1000)$, water $(0)$ and skeletal muscle (-29 to 150) as previously described $[28,29]$. Muscle attenuation (MA) was determined as the average HU for each scan. Total CSA of skeletal muscle was normalized by dividing by squared height $\left(\mathrm{m}^{2}\right)$ and expressed as a skeletal muscle index (SMI) $\left(\mathrm{cm}^{2} / \mathrm{m}^{2}\right)$. MLBW was calculated using the total muscle area $\left(\mathrm{cm}^{2}\right)$ from each CT scan [20]:
$\operatorname{MLBW}(\mathrm{kg})=\left(L 3\left(\mathrm{~cm}^{2}\right) \times 0.3\right)+6.06$

\section{Results}

\subsection{Patient Demographics}

This study was conducted using datasets from two clinical trials with REC-2282 at The Ohio State University Comprehensive Cancer Center. Combined, OSU09102 (NCT01129193), the first-in-human trial, and OSU11130 (NCT01798901) included a total of 57 patients with relapsed or refractory malignancies. Summaries of the patient demographics and disease characteristics were previously published, and a subset of patient features are also presented in Supplemental Table 2 [2, 4, 5, 17]. Anthropometric measurements were not available for one patient. Demographic and body size factors for all patients are summarized in Table 2. Doses of REC-2282 are summarized in Table 1.

\subsection{Population Pharmacokinetic Model for REC-2282}

A total of 882 REC-2282 plasma concentrations were obtained from 55 subjects. Forty-four (44) subjects had day 1 and either day 5 or day 19 plasma concentrations. The
Table 2 Summary of patient characteristics from clinical trials

\begin{tabular}{llll}
\hline Characteristic & & All patients & Patients with CT scans \\
\hline No. & & 56 & 21 \\
WT $(\mathrm{kg})$ & Mean (SD) & $76.5(17.8)$ & $78.6(14.9)$ \\
& Median (Min-Max) & $76.1(42.9-122.4)$ & $78.1(42.9-102.6)$ \\
Sex & Male (\%) & $27(48.2)$ & $12(57.1)$ \\
& Female (\%) & $29(51.8)$ & $9(42.9)$ \\
FFM $(\mathrm{kg})$ & Mean (SD) & $51.4(12.3)$ & $54.6(8.9)$ \\
& Median (min-max) & $50.8(29.9-76.0)$ & $54.8(37.2-68.5)$ \\
AGE $(\mathrm{years})$ & Mean (SD) & $60.0(14.1)$ & $59.4(12.4)$ \\
& Median (min-max) & $63(20-80)$ & $58(33-80)$ \\
LBW $(\mathrm{kg})$ & Mean (SD) & $54.9(11.6)$ & $57.7(8.5)$ \\
& Median (min-max) & $53.1(34.2-79.8)$ & $56.5(42.1-71.8)$ \\
BSA $\left(\mathrm{m}^{2}\right)$ & Mean (SD) & $1.9(0.3)$ & $1.9(0.2)$ \\
BMI $\left(\mathrm{kg} / \mathrm{m}^{2}\right)$ & Median (min-max) & $1.9(1.4-2.4)$ & $1.9(1.3-2.2)$ \\
& Mean (SD) & $26.7(5.4)$ & $27.8(5.1)$ \\
SMI $\left(\mathrm{cm}^{2} / \mathrm{m}^{2}\right)$ & Median (min-max) & $26.0(18.5-43.6)$ & $26.8(18.5-39.7)$ \\
MA $(\mathrm{HU})$ & Mean (SD) & NA & $44.71(11.1)$ \\
Missing & Mean (SD) & NA & $31.5(10.0)$ \\
\hline
\end{tabular}

One patient was treated at Nationwide Children's Hospital and therefore did not have accessible anthropometric data

$W T$ patient body weight, $F F M$ fat-free mass, $L B W$ lean body weight, $B S A$ body surface area, $B M I$ body mass index, $S M I$ skeletal muscle index, $M A$ muscle attenuation, $H U$ Hounsfield units, $S D$ standard deviation, $N A$ not available, $C T$ computed tomography 

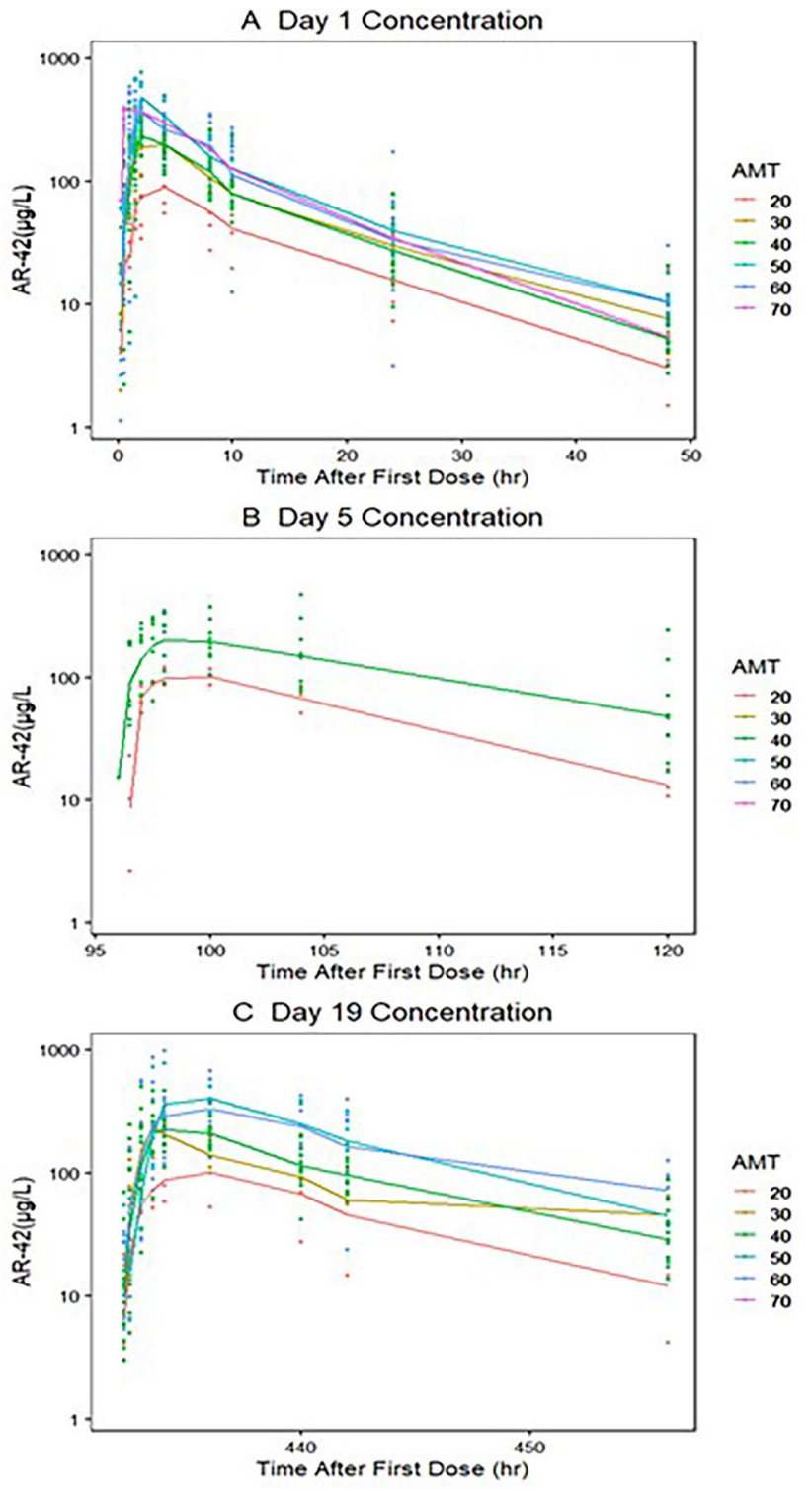

Fig. 1 Concentration vs. time plots of REC-2282 (AR-42) at A day 1; B day 5; C day 19. AMT Dose levels of AR-42 (mg). Dots represent observed concentrations while lines represent the mean concentrations of the dose group

observed plasma concentration-time data for all 55 subjects can be seen in Fig. 1. Several models were evaluated, including one- and two-compartment models without and with lag time, and also without and with transit compartments to accommodate observed variability in apparent lag time. A two-compartment base model with one transit compartment for absorption, lag time, first-order elimination and a proportional error model best described the data (see Fig. 2). The model was parameterized in terms of clearance (CL), volume of distribution of the central compartment $(\mathrm{Vc})$, inter-compartmental clearance $(\mathrm{Q})$, volume of distribution of the peripheral compartment $(\mathrm{Vp})$, absorption rate constant (ka) and ALAG. IIV was estimated for CL and ka with sufficiently low shrinkage $(<15 \%)$. IIV was estimated for ALAG but with high shrinkage (62.5\%). Base model parameters are displayed in Table 3, and relevant diagnostic plots can be found in Supplemental Figure 1.

For covariate analysis, each covariate was evaluated individually on each model parameter, and those that achieved significance $(P<0.05)$ were evaluated within the model in multivariate analysis using a step-wise selection procedure with forward addition and backward elimination. FFM, LBW, BSA, height, weight, tumor type and sex were significant covariates on CL, and tumor type was significant on ALAG in univariate analysis (see Table 4). Multivariate analysis was challenging because of instability of the model and inability to obtain covariance estimates for most models attempted. We ultimately chose to remove IIV on the ALAG parameter, which improved model stability. Interestingly, we observed improved model fit when the categorical covariates, tumor type and formulation were included on ALAG without IIV. In the final model FFM was retained as a single covariate on $\mathrm{CL}$, and tumor type and formulation were retained as covariates on ALAG. The final model presented acceptable estimation errors of the parameters $(<$ $40 \%$ ) except for tumor type (60.6\%) and formulation (50\%); shrinkages were sufficiently low $(<16 \%)$, while random error was high (54.5\%). The detailed parameter estimates in the structural and final covariate model are listed in Table 3.

Bias and stability of the final model were assessed by evaluating $95 \%$ confidence intervals of predicted parameters utilizing the bootstrap method (Table 3). One thousand resampled datasets were simulated to evaluate prediction performance of the final model by VPC, in which observed data were compared with the $95 \%$ confidence intervals of the predicted values (Fig. 3). Overall, the final model sufficiently described the observed concentrations; however, the model may fail to adequately capture the $C_{\max }$ in some patients.

\subsection{Analysis of Patient Body Composition}

Of the 56 patients, 21 had a CT image during cycle 1 evaluable for skeletal muscle area (SMA), SMI and MA at the L3 landmark. Anthropometric measurements and body composition parameters for patients with evaluable CT scans are summarized in Table 2. The mean \pm SD SMI $\left(\mathrm{cm}^{2} / \mathrm{m}^{2}\right)$ and MA (HU) for L3 were $44.71 \pm 11.1$ and $31.5 \pm 10$, respectively. Patients presented with a variable range of BSA, weight, BMI, SMI and MA.

As an alternative to traditional anthropometric-derived body descriptors (FFM, LBW, BSA, etc.), MLBW and MA were evaluated as continuous variables in a subset covariate analysis with the 21 patients characterized above. When MLBWs were calculated and compared to 


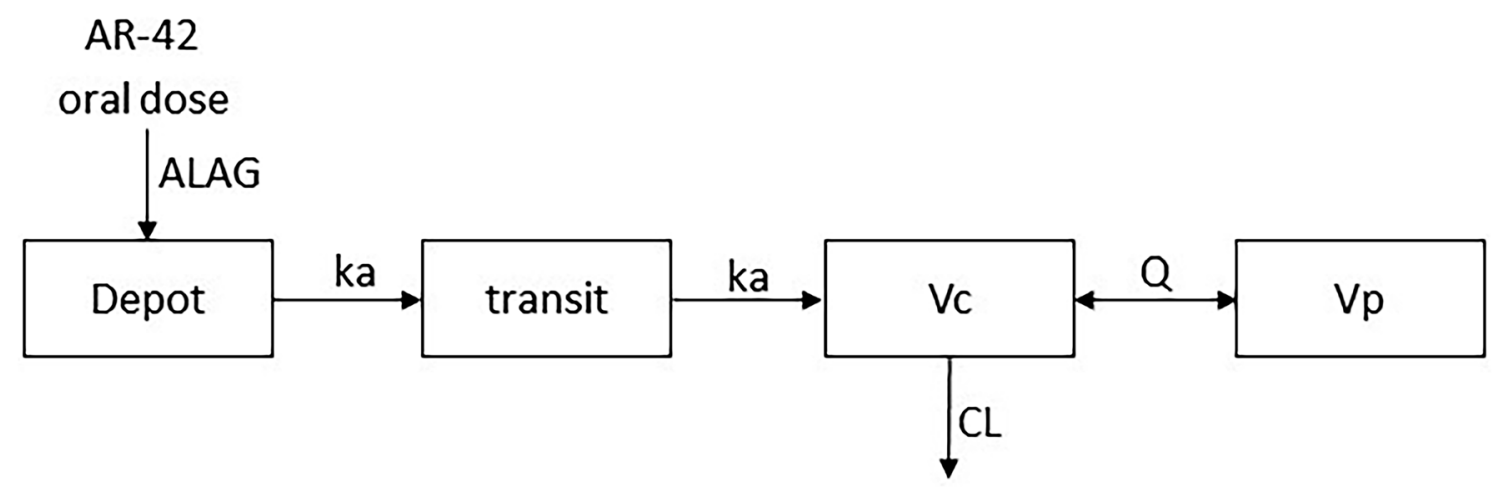

Fig. 2 A two-compartmental structural model describing the pharmacokinetics of REC-2282 (AR-42). $A L A G$ absorption lag time, $k a$ absorption rate constant, $V c$ volume of central compartment, $C L$

anthropomorphic-derived LBW and FFM, MLBWs were consistently lower (Supplemental Figure 2). A subset univariate analysis with patients with CT scans revealed anthropomorphic-derived LBW as a significant covariate in this subset of patients, but there were no further observable trends between the individual parameter estimates and any of the other covariates evaluated.

\subsection{Simulation of Flat vs. Body Size-Normalized Dosing}

To further evaluate whether body size normalization of the REC-2282 dose could decrease inter-subject variability, simulations were performed to compare the difference between flat dose-based simulation and body size-normalized simulation. AUCs were simulated from a subset of patients who were administered with a 40-mg flat dose three times weekly, the same subset of patients but with a LBW-based dose, a LBW dose based on the available capsule strengths or a BSA-based dose. The 40-mg dose level was selected since this dose level contained the greatest number of patients with evaluable pharmacokinetic profiles. Simulations showed no differences in patient AUCs between these simulated dosing regimens, though differences in standard deviations were observed with the flat dose having the smallest inter-subject variability for simulated AUCs (Supplemental Figure 3). Given the lack of difference observed in simulated AUC and the higher AUC variability among the simulated body size-normalized dose regimens compared to the flat dose regimen, we concluded the flat dose method was in fact the most appropriate choice based on the current dataset. apparent clearance, $Q$ inter-compartmental clearance, $V p$ volume of peripheral compartment

\section{Discussion}

REC-2282 is in clinical development for treatment of cancer and neurofibromatosis. Thus far, five clinical trials have been initiated in solid tumor and hematologic malignancies and in vestibular schwanommas. Two of these trials were completed, and three are either on hold (2) or were terminated (1) as the new drug owner, Recursion Pharmaceuticals, establishes new clinical drug supply. Overall, REC2282 has demonstrated acceptable safety in these early phase studies, and promising clinical activity has been observed in some patients, especially in those with schwanomas [3, 30]. Notably, REC-2282 was also discovered through novel in silico screening to have high activity in NF2-driven tumors [5], which has motivated investigators to further explore this area clinically.

In this report, we combined data from two of the completed trials, including the first-in-human study, to characterize pharmacokinetics of REC-2282 across a population of 55 patients with both solid and hematologic cancers and across a dose range from $20 \mathrm{mg}$ to $80 \mathrm{mg}$. Our analysis revealed high variability in REC-2282 exposure, with dose-normalized AUC and $C_{\max }$ of $24.1 \%$ and $29.6 \%$, respectively. The data also demonstrated REC-2282 pharmacokinetics is doseproportional across this range of flat doses. Using nonlinear mixed effects modeling, we explored a variety of structural models and performed covariate analysis to identify factors that could explain the significant observed variability. Our final model included a single transit compartment for oral absorption with ka, ALAG, a central plasma compartment and a peripheral tissue compartment. FFM was the only significant covariate identified on CL, and it described only a small portion of the overall observed pharmacokinetic variability (2.6\% of IIV on apparent clearance).

Despite a large portion of the variability unexplained by the available covariate data, the model performed well in describing the data overall, though one obvious limitation 


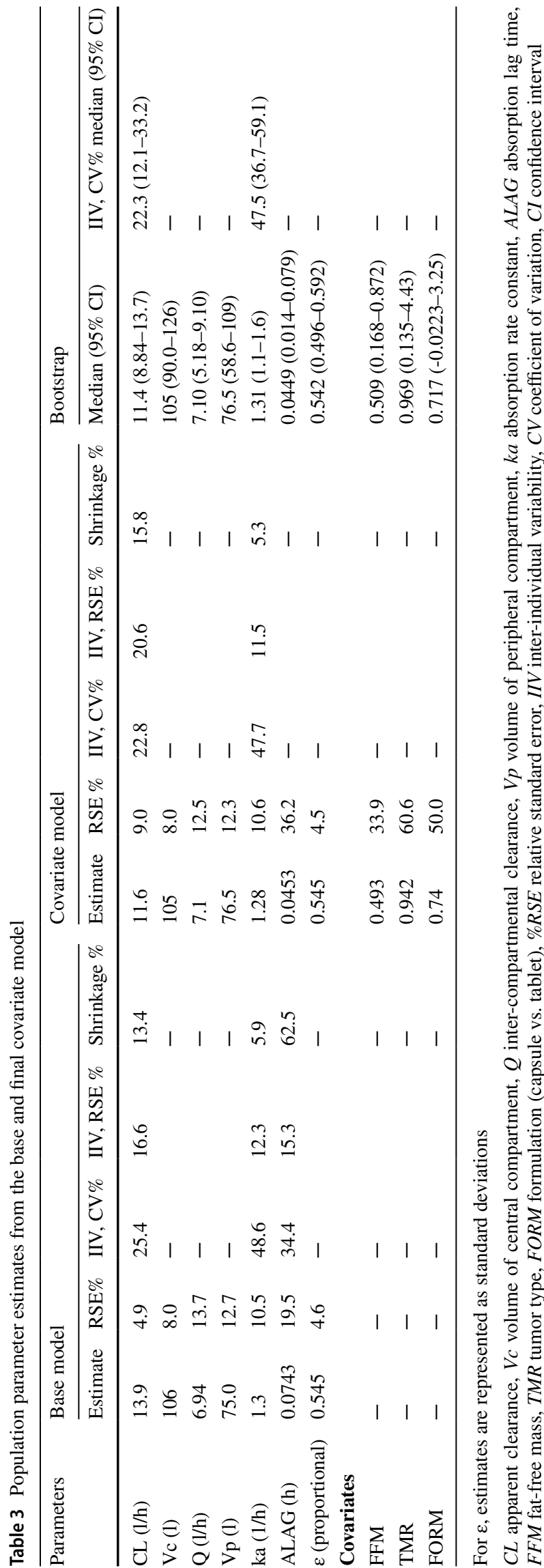

Table 4 Results of univariate covariate analysis

\begin{tabular}{llll}
\hline Univariate analysis & \multicolumn{3}{l}{$\begin{array}{l}\text { Univariate covariate analysis in } \\
\text { population model }\end{array}$} \\
\cline { 2 - 4 } & OFV & $\Delta$ OFV & $P$ value \\
\hline 1. Base model & 26.451 & - & - \\
2. 1 + FORM on ALAG & 24.537 & -1.91 & NS \\
3. 1 + Tumor on ALAG & 22.320 & -4.13 & $<0.05$ \\
4. 1 + Weight on CL & 20.693 & -5.76 & $<0.01$ \\
5. 1 + BSA on CL & 19.445 & -7.01 & $<0.01$ \\
6. 1 + Height on CL & 18.585 & -7.87 & $<0.01$ \\
7. 1 + LBW on CL & 17.994 & -8.46 & $<0.01$ \\
8. 1 + SEX on CL & 17.733 & -8.72 & $<0.01$ \\
9. 1 + FFM on CL & 17.429 & -9.02 & $<0.01$ \\
\hline
\end{tabular}

Covariates that met significance are denoted by $(P<0.05)$ or $(P<0.01)$

FORM formulation (capsule vs. tablet), $B S A$ body surface area, $L B W$ lean body weight, $F F M$ fat-free mass, $O F V$ objective function value, $\triangle O F V$ difference of objective function value

was its inability to capture the time of peak concentration $\left(T_{\max }\right)$. Our model consistently over-predicted $T_{\max }$, which we sought to address by incorporating first-order, zeroorder and parallel zero- and first-order oral absorption with or without lag time or transit compartments. However, the model became over-parameterized when adding multiple parameters to describe the absorption process. Additionally, although IIV on ka or other absorption parameters significantly improved the $T_{\max }$ predictions, this strategy resulted in high shrinkage of the IIV terms. These results suggested a significant portion of the observed IIV was due to variability in absorption. Notably, patients in these two trials were fasted prior to drug administration, which allowed us to rule out variability due to differences in food intake among enrolled patients. As we were not able to achieve acceptable parameter precision or model stability when we attempted to model various types of absorption processes, we ultimately settled on the single transit compartment model with firstorder absorption, where a majority of IIV remained. In this final model, we added a lag time parameter into the transit compartment and identified tumor type and formulation as significant covariates on this parameter. Given the difference in sampling schemes, tumor type may be attributed to a study effect between these two trials. Further consideration of alternate oral absorption models, adjusted sampling schemes to better describe the absorption process and the assessment of REC-2282 bioavailability with pharmacokinetic data obtained after intravenous dosing in future clinical trials may be warranted.

During our search for covariates to describe the observed pharmacokinetic variability, and because we wanted to determine whether flat dosing vs. body size-normalized 


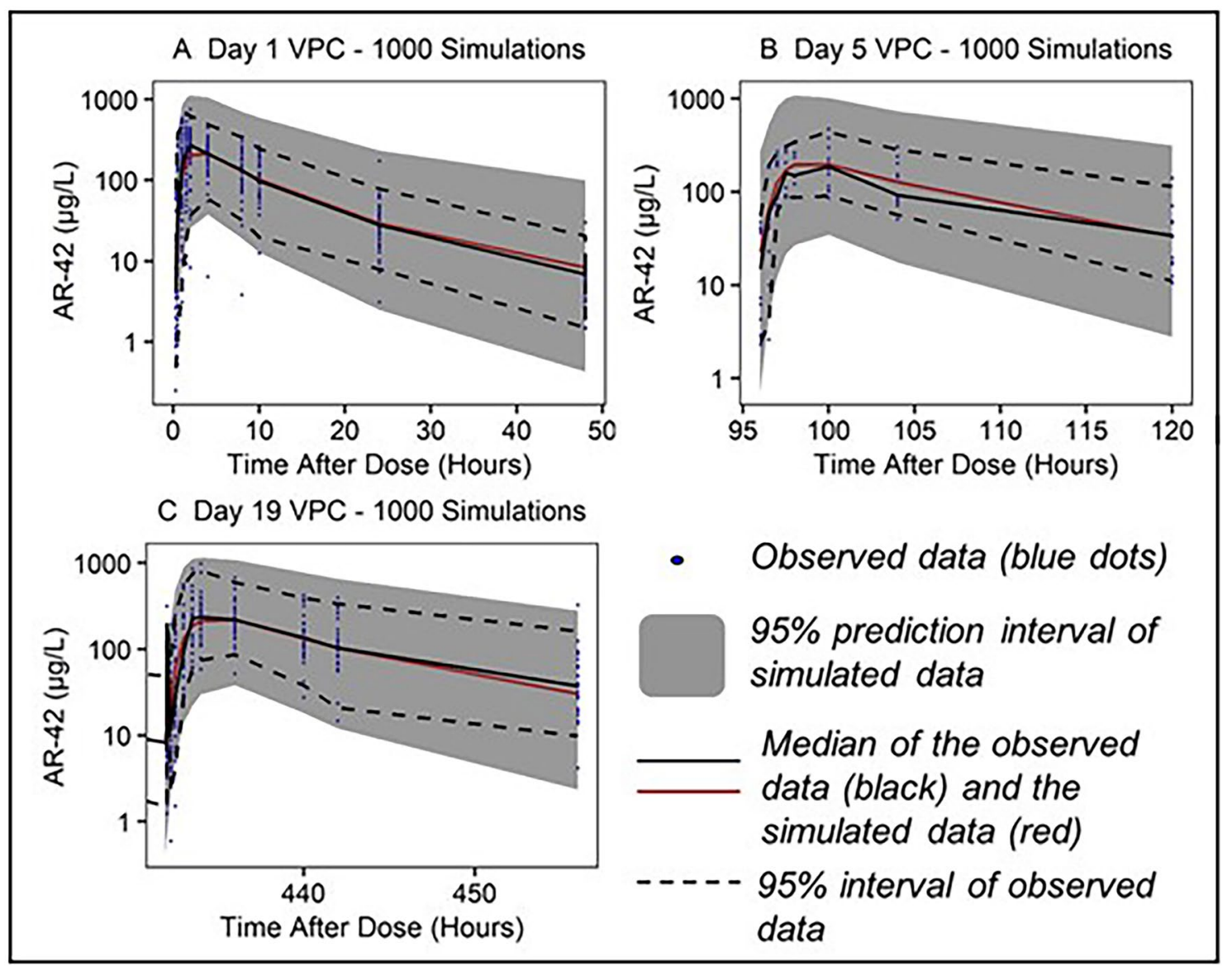

Fig. 3 Visual predictive check (VPC) evaluating the final model of REC-2282 (AR-42) pharmacokinetic on Days 1 (A), 5 (B) and 19 (C)

dosing was appropriate for clinical development, we interrogated various body size descriptors including anthropomorphic-derived LBW, FFM, BMI and CT-derived lean mass (MLBW) using SMI and MA to determine whether these improved our ability to describe REC-2282 pharmacokinetic variability. Notably, in the subset of patients with CT scans, we observed lower estimates of lean mass by CT compared to LBW or FFM (Supplemental Figure 3). This finding is consistent with other reports directly comparing imaging-based assessments of lean body mass with anthropomorphic-derived parameters [31] and suggests that cancer patients' lean body mass may be consistently over estimated by traditional equations relating anthropomorphic features to lean body mass. Multiple reports suggest reduced LBM in cancer patients is associated with increased therapeutic toxicity [32,33]. To this end, in the subset of patients with evaluable CT images, we observed patients with higher SMI completed greater than one cycle of REC-2282 compared to those completing one or less cycles (Supplemental Figure 4). However, in a subset covariate analysis we did not see a major impact from SMI or MA on IIV. Ultimately none of the other body size descriptors we evaluated provided an improved understanding of variability in our model. Of note, we observed slightly higher (15\%) AUCs in females vs. males. With this, we chose to evaluate the relationship between observed dose-normalized AUC vs. FFM and observed a weak association $\left(R^{2}=0.18\right)$. Although this relationship was significant $(P<0.01)$, the portion of observed variability described by FFM was very low $(2.6 \%)$. This exhaustive analysis of body size factors as potential covariates and the ability of only one of these factors to explain only a very small (2.6\%) portion of observed pharmacokinetic variability support the decision for flat dosing instead of body size-normalized dosing as the appropriate choice for clinical development.

\section{Conclusion}

REC-2282 is a promising new therapeutic with the potential to treat neurofibromatosis type 2 , a devastating disease currently lacking effective treatment options [34]. This first population pharmacokinetic analysis demonstrates high pharmacokinetic variability, which may be a function of 
variable oral absorption. Future modeling of REC-2282 absorption is warranted, though it will likely require additional data to understand the contributions of formulation, food effects, drug transport, etc. Our analysis suggested FFM does explain portions of IIV; however, like many oral drugs, the choice of flat dosing is appropriate, and little or nothing would be gained by utilizing body size-normalized dosing.

Supplementary Information The online version contains supplementary material available at https://doi.org/10.1007/s13318-021-00722-z.

Acknowledgments REC-2282 (AR-42) was provided by Arno Therapeutics, Inc. We thank the laboratory of Vickie Baracos for providing us vital training in their CT imaging methodology.

\section{Declarations}

Funding This project was supported by The Ohio State University Comprehensive Cancer Center (P30CA016058); National Cancer Institute K12CA133250 (Coss) and R01CA201382 (Phelps); Eli Lilly Graduate Student Fellowship (Liva).

Conflict of Interest CCC holds intellectual property concerning the use of REC-2282 (AR-42) and has received royalties associated with the licensing of this intellectual property to Recursion Pharmaceuticals. $\mathrm{CCC}$ has no direct financial relationship with Recursion Pharmaceuticals, and Recursion Pharmaceuticals did not contribute to this work in any way. $\mathrm{CCH}$ has received research grants from Oncolytics Biotech, research and personal grants from Janssen, BMS, Sanofi. Nektar, and Karyopharm, and personal grants from Imbrium and Oncopeptides over the last 24 months all outside the submitted work.

Ethics Approval All procedures in this study were in accordance with the 1964 Helsinki declaration (and its amendments) and the details of the Ethics Committee or institutional review board which approved the studies.

Consent to participate Not applicable.

Consent for Publication Not applicable.

Availability of data and material All data are included in the manuscript.

Code availability Not applicable.

Authors' Contributions $\mathrm{AM}, \mathrm{AW}, \mathrm{KD}, \mathrm{CH}$ designed the clinical trials, accrued patients to the studies, and supported data acquisition, data analysis, and drafting of the manuscript; MP and CC directed the data analysis and supported drafting and editing of the manuscript; SL, MC and JW generated the PK data, performed the data analyses, drafted and edited the manuscript.

Open Access This article is licensed under a Creative Commons Attribution-NonCommercial 4.0 International License, which permits any non-commercial use, sharing, adaptation, distribution and reproduction in any medium or format, as long as you give appropriate credit to the original author(s) and the source, provide a link to the Creative Commons licence, and indicate if changes were made. The images or other third party material in this article are included in the article's Creative Commons licence, unless indicated otherwise in a credit line to the material. If material is not included in the article's Creative Commons licence and your intended use is not permitted by statutory regulation or exceeds the permitted use, you will need to obtain permission directly from the copyright holder. To view a copy of this licence, visit http://creativecommons.org/licenses/by-nc/4.0/.

\section{References}

1. Shah RR. Safety and tolerability of histone deacetylase (HDAC) inhibitors in oncology. Drug Saf. 2019;42(2):235-45.

2. Sborov DW, Canella A, Hade EM, et al. A phase 1 trial of the HDAC inhibitor AR-42 in patients with multiple myeloma and T- and B-cell lymphomas. Leuk Lymphoma. 2017;58(10):2310-8.

3. Burns SS, Akhmametyeva EM, Oblinger JL, et al. Histone deacetylase inhibitor AR-42 differentially affects cell-cycle transit in meningeal and meningioma cells, potently inhibiting NF2deficient meningioma growth. Cancer Res. 2013;73(2):792-803.

4. Collier KA, Valencia $\mathrm{H}$, Newton $\mathrm{H}$, et al. A phase 1 trial of the histone deacetylase inhibitor AR-42 in patients with neurofibromatosis type 2-associated tumors and advanced solid malignancies. Cancer Chemother Pharmacol. 2021;87(5):599-611.

5. Valencia H, Newton HB, Hade E, et al. A phase 1 study of AR-42 in patients with advanced solid tumors, including nervous system tumors. J Clin Oncol. 2016;34(15_suppl):2558-2558.

6. Alfa R. Artificial intelligence lights a beacon to new medicine for neurofibromatosis type 2. In. Recursion. https://medium.com/ recursion-pharmaceuticals/artificial-intelligence-provides-a-beacon-to-new-medicine-for-neurofibromatosis-type-2-d0ec41344f 50. Accessed 22 Sep 2021.

7. Cheng H, Xie Z, Jones WP, et al. Preclinical pharmacokinetics Study of R- and S-enantiomers of the histone deacetylase inhibitor, AR-42 (NSC 731438), in rodents. AAPS J. 2016;18(3):737-45.

8. Undevia SD, Gomez-Abuin G, Ratain MJ. Pharmacokinetic variability of anticancer agents. Nat Rev Cancer. 2005;5(6):447-58.

9. Hopkins JJ, Sawyer MB. A review of body composition and pharmacokinetics in oncology. Expert Rev Clin Pharmacol. 2017;10(9):947-56.

10. Baracos VE, Reiman T, Mourtzakis M, et al. Body composition in patients with non-small cell lung cancer: a contemporary view of cancer cachexia with the use of computed tomography image analysis. Am J Clin Nutr. 2010;91(4):1133S-1137S.

11. Baker SD, Verweij J, Rowinsky EK, et al. Role of body surface area in dosing of investigational anticancer agents in adults, 19912001. J Natl Cancer Inst. 2002;94(24):1883-8.

12. Chu MP, Li Y, Ghosh S, et al. Body composition is prognostic and predictive of ipilimumab activity in metastatic melanoma. $\mathrm{J}$ Cachexia Sarcopenia Muscle. 2020;11(3):748-55.

13. Sparreboom A, Wolff AC, Mathijssen RH, et al. Evaluation of alternate size descriptors for dose calculation of anticancer drugs in the obese. J Clin Oncol. 2007;25(30):4707-13.

14. Morgan DJ, Bray KM. Lean body mass as a predictor of drug dosage. Implications for drug therapy. Clin Pharmacokinet. 1994;26(4):292-307.

15. Bins S, Ratain MJ, Mathijssen RH. Conventional dosing of anticancer agents: precisely wrong or just inaccurate? Clin Pharmacol Ther. 2014;95(4):361-4.

16. Miller AA. Body surface area in dosing anticancer agents: scratch the surface! J Natl Cancer Inst. 2002;94(24):1822-3.

17. Liva SG, Coss CC, Wang J, et al. Phase I study of AR-42 and decitabine in acute myeloid leukemia. Leuk Lymphoma. 2020;61(6):1484-92.

18. Bauer RJ. Nonmem users guide introduction to Nonmem 7.3.0. In: ICON Development Solutions; 2015.

19. Savic RM, Jonker DM, Kerbusch T, et al. Implementation of a transit compartment model for describing drug absorption 
in pharmacokinetic studies. J Pharmacokinet Pharmacodyn. 2007;34(5):711-26.

20. Mourtzakis M, Prado CM, Lieffers JR, et al. A practical and precise approach to quantification of body composition in cancer patients using computed tomography images acquired during routine care. Appl Physiol Nutr Metab. 2008;33(5):997-1006.

21. Bergstrand M, Hooker AC, Wallin JE, et al. Prediction-corrected visual predictive checks for diagnosing nonlinear mixed-effects models. AAPS J. 2011;13(2):143-51.

22. Cheymol G. Effects of obesity on pharmacokinetics implications for drug therapy. Clin Pharmacokinet. 2000;39(3):215-31.

23. Janmahasatian S, Duffull SB, Ash S, et al. Quantification of lean bodyweight. Clin Pharmacokinet. 2005;44(10):1051-65.

24. Zierle-Ghosh A, Jan A. Physiology, body mass index. Treasure Island: StatPearls; 2020.

25. Du Bois D, Du Bois EF. A formula to estimate the approximate surface area if height and weight be known. 1916. Nutrition. 1989;5(5):303-11 (discussion 312-3).

26. Shen W, Punyanitya M, Wang Z, et al. Total body skeletal muscle and adipose tissue volumes: estimation from a single abdominal cross-sectional image. J Appl Physiol (1985). 2004;97(6):2333-8.

27. Shen W, Punyanitya M, Wang Z, et al. Visceral adipose tissue: relations between single-slice areas and total volume. Am J Clin Nutr. 2004;80(2):271-8.
28. Heymsfield SB, Smith R, Aulet M, et al. Appendicular skeletal muscle mass: measurement by dual-photon absorptiometry. Am J Clin Nutr. 1990;52(2):214-8.

29. Dennis RA, Long DE, Landes RD, et al. Tutorial for using SliceOmatic to calculate thigh area and composition from computed tomography images from older adults. PLoS ONE. 2018;13(10): e0204529.

30. Bush ML, Oblinger J, Brendel V, et al. AR42, a novel histone deacetylase inhibitor, as a potential therapy for vestibular schwannomas and meningiomas. Neuro Oncol. 2011;13(9):983-99.

31. Moisey LL, Mourtzakis M, Kozar RA, et al. Existing equations to estimate lean body mass are not accurate in the critically ill: results of a multicenter observational study. Clin Nutr. 2017;36(6):1701-6.

32. Prado CM, Baracos VE, McCargar LJ, et al. Body composition as an independent determinant of 5-fluorouracil-based chemotherapy toxicity. Clin Cancer Res. 2007;13(11):3264-8.

33. Ali R, Baracos VE, Sawyer MB, et al. Lean body mass as an independent determinant of dose-limiting toxicity and neuropathy in patients with colon cancer treated with FOLFOX regimens. Cancer Med. 2016;5(4):607-16.

34. Plotkin SR, Wick A. Neurofibromatosis and Schwannomatosis. Semin Neurol. 2018;38(1):73-85. 